médecine/sciences $1988 ; 4: 168-178$

\title{
Une malformation fotale à type de sirène : un modèle pour la tératologie
}

L'étude d'une sirène s'étant développée jusqu'à sept mois de gestation permet de proposer un modèle des possibles mécanismes des anomalies du développement embryonnaire. L'embryogenèse implique une succession d'informations transmises par des cellules, à un stade précis de leur différenciation, à d'autres cellules dont elles déterminent le destin. De ce fait, une anomalie dans la différenciation d'une structure donnée, que sa cause soit génétique ou non, pourra perturber le destin de toutes les cellules dont la différenciation dépend d'influences postérieures à l'expression de l'anomalie.

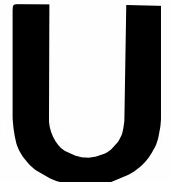

ne équipe d'anatomistes de la faculté de médecine de Marseille, dirigée par le professeur $\mathrm{J}$. Bonnoît, a eu le privilège assez rare de faire l'autopsie d'une sirène (figure $1 A$ ) qui s'est développée jusqu'au septième mois de la gestation [1]. Rappelons que la sirénomélie est caractérisée par la fusion des membres pelviens, accompagnée par le rejet des rotules en position latérale et par l'orientation des pieds vers l'arrière.

2. Byrne J, Warburton D, Kline J, Blanc W, Stein Z. Morphology of early fetal deaths and their chromosomal characteristics. Teratology $1985 ; 32: 297-315$

3. Chandebois R, Brunet C. The origin of abnormality in a human simelian foetus as elucidated by our knowledge of vertebrate development. Teratology 1987 ; 36 : 11-22.

4. L'Héritier P. Génétique. Paris: Masson, 1975

5. Lewin R. Why is development so illogical ? Science $1984 ; 224$ : 1327-9.

6. Gurdon JB. Embryonic induction : molecular prospects. Development $1987 ; 99: 285$ 306.

\section{ADRESSE}

R. Chandebois : laboratoire de morphogénétique animale, université de Provence, centre Saint-Charles, place Victor-Hugo, 13331 Mar-

\section{Malformations mais caryotype normal}

Chez ce foetus, la région thoracique a été la seule épargnée. La sirénomélie est aggravée par une atrophie distale: les deux fémurs s'articulent avec un seul tibia dans le prolongement duquel se trouvent un métatarsien et un gros orteil (figure 1B). A la première vertèbre sacrée fait directement suite une file de petites vertèbres (vraisemblablement un coccyx atypique), repliée vers l'extrémité crâniale. Le segment terminal de la moelle épinière se situe au niveau normal, mais il est effilé et les nerfs de la queue de cheval sont en nombre réduit. L'intestin se termine en cul-de-sac au niveau du côlon descendant. Il n'y a ni vessie ni reins. Toutefois des tubules urinifères d'aspect embryonnaire ont été trouvés dans deux kystes pelviens, l'un d'eux enveloppé par de l'épithélium intestinal. L'orientation des muscles abdominaux est faussée. La tête est dystrophique : la boîte crânienne est exagérément développée selon l'axe fronto-occipital ; les fosses nasales sont trop larges, les oreilles en position basse.

L'examen du caryotype n'a révélé aucune aberration, ni dans le nombre ni dans la structure des chromosomes.

\section{Problème de génétique ou d'embryologie?}

Les données que nous possédons actuellement ne permettent plus de croire à une relation obligatoire entre la sirénomélie et une anomalie génétique. En effet, un cas de trisomie 21 a été signalé chez les sirènes alors que leur caryotype paraît habituellement normal. Mais cette même trisomie 21 a été trouvée chez des foetus présentant à la fois des membres courts et une dystrophie 


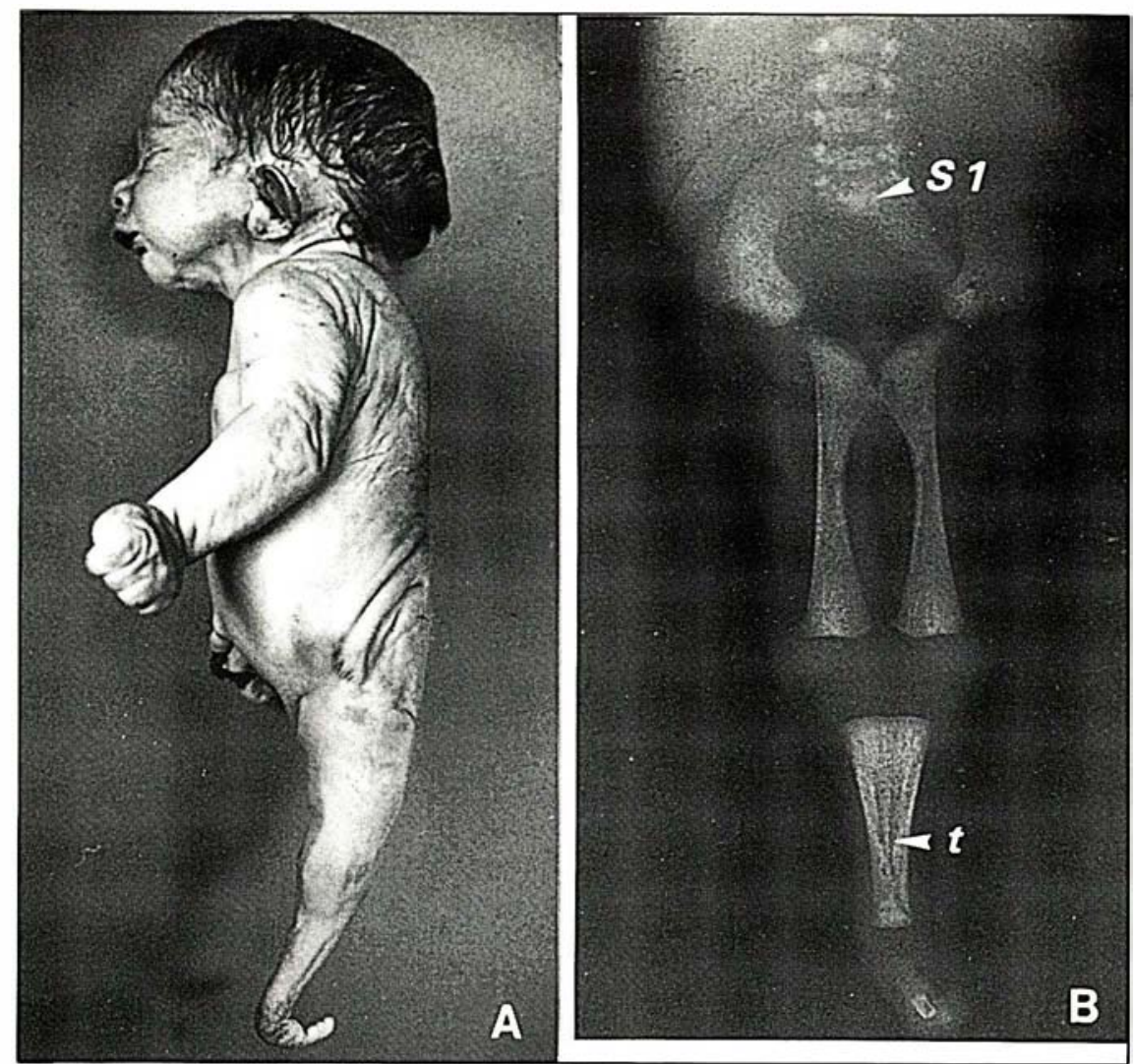

Figure 1. La sirène. A. Profil gauche de la sirène. On remarquera plus particulièrement l'atrophie du pied, la position de la rotule, la dystrophie de la boîte crânienne, l'implantation basse de l'oreille. B. Radiographie de la région pelvienne et du membre postérieur. Le rachis est normal en avant de la première vertèbre sacrée (S1). On note la soudure des tibias (t), celle des métatarsiens et des doigts I. Les péronés et les rayons /I et $N$ de l'autopode ne sont pas formés. (Clichés: J. Bonnoît.)

céphalique, de même que chez des embryons normaux [2]. Très probablement, la cause première de la sirénomélie n'est pas la même dans tous les cas et elle n'est pas nécessairement d'origine génétique. Dans tous les cas, néanmoins, le même type d'accident se produit dans la construction de l'organisme et c'est lui qu'il importe de découvrir.

Toutes les malformations observées chez la sirène, dont il est ici question, évoquent les résultats de nombreuses interventions chirurgicales réalisées sur des embryons d'amphibiens, d'oiseaux et de mammifères - celles$m / s n^{\circ} 3$ vol. 4, mars 88 cription et de la synthèse des protéines. Divers auteurs ont souligné la nécessité de prendre en considération la communication cellulaire $[4,5]$. Pour que cela soit possible, il faudrait cependant que les théories classiques de l'embryologie nous en donnent une vue globale et cohérente, ce qui est loin d'être le cas. L'émergence des tissus est expliquée par les phénomènes d'induction. Les mécanismes cellulaires et moléculaires qu'on leur attribue actuellement cadrent mal avec de nombreuses données récentes [6]. En outre, ces phénomènes sont tenus pour indépendants de la création et du maintien des structures visibles [7], généralement attribués à des gradients de «morphogènes ». $\mathrm{Si}$ on reconsidère l'ensemble de nos acquis expérimentaux aux niveaux organique, cellulaire et moléculaire, il apparaît que la morphogenèse découle de la progression de la différenciation tissulaire, tandis que les gradients y jouent un rôle secondaire [8]. Un concept unitaire du développement a été finalement proposé [9]. Il a été appelé «sociologie cellulaire » pour bien souligner que la complication progressive de l'organisation a pour principe une communication incessante entre les cellules, dont celles-ci conservent la «mémoire» collectivement (en partie individuellement). De ce concept se dégage un schéma concret de l'expression des gènes dans les structures visibles [10] qu'illustre parfaitement le développement de notre sirène.

là mêmes qui ont contribue progrès les plus décisifs dans la connaissance des interactions cellulaires impliquées dans la morphogenèse des vertébrés. De ce fait, il a été possible de reconstituer le développement du fœetus et de situer les causes possibles de la monstruosité.

Les résultats de cette analyse ont été publiés dans la littérature [3]. Dans cet article, nous voulons insister particulièrement sur leur intérêt théorique. En effet, le problème de l'expression des gènes dans les structures visibles est encore généralement ramené à celui de la régulation de la trans-

\section{Comportement social des cellules}

La différenciation tissulaire qui implique la transformation progressive des activités spécifiques des cellules et leur diversification par dichotomies successives - débute avec la segmentation de l'œuf. Son principe moteur est la « progression autonome» (PA). Si une population de cellules embryonnaires est isolée de ses voisines, elle continue à évoluer pendant un certain 


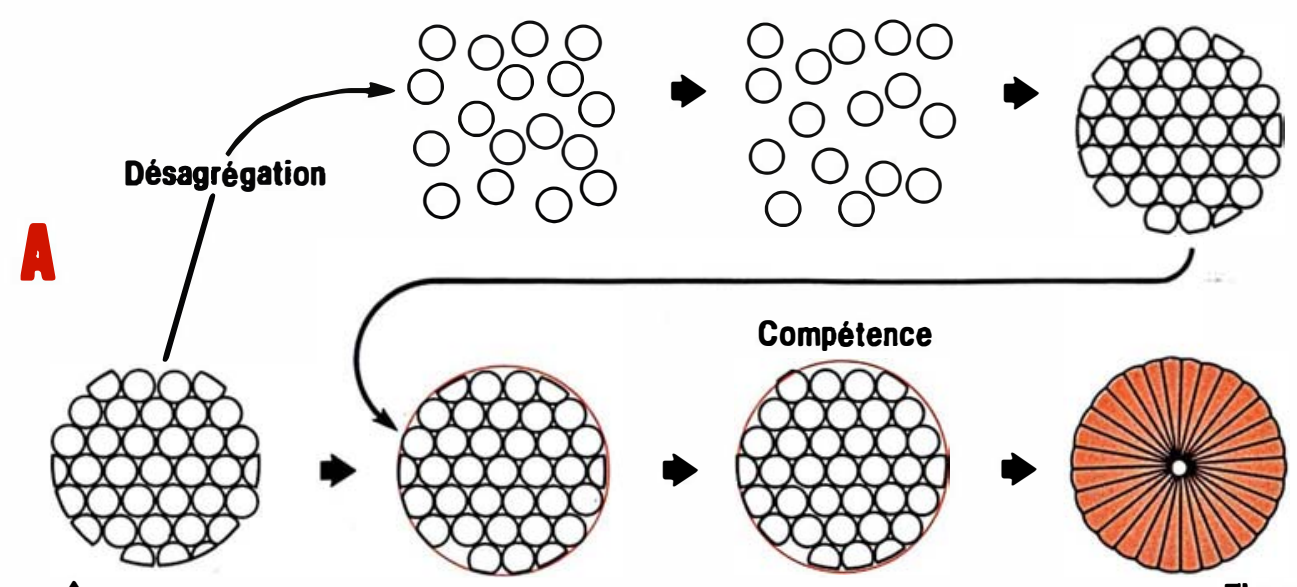

个Déterm.1

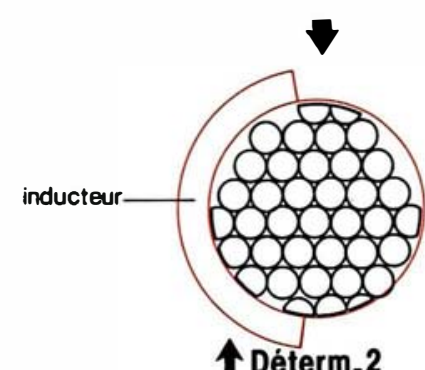

A Determ.2

Tissu 1

8

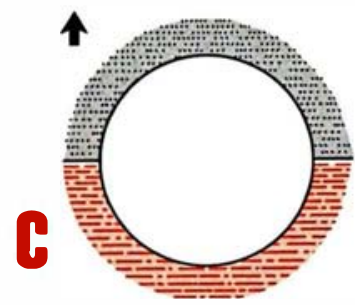

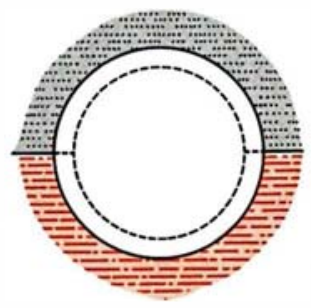

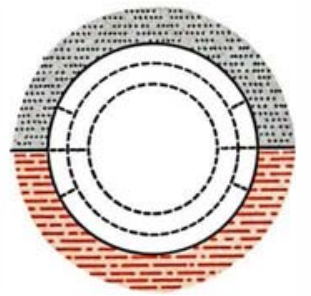

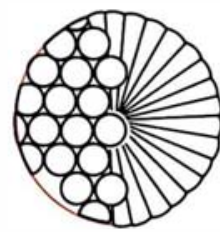

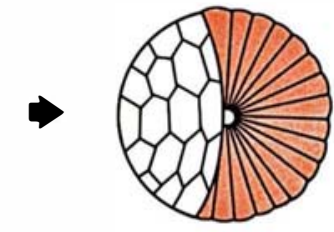

Tissu 2

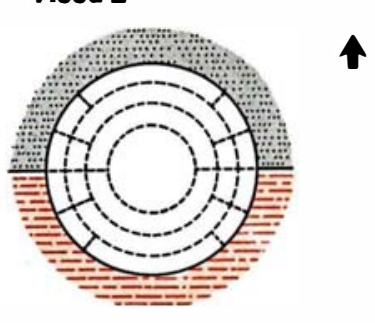

Figure 2. Schémas résumant les mécanismes de l'organogenèse chez les animaux pluricellulaires. A. La progression autonome. Isolée, une population de cellules continue à évoluer pendant un certain temps, ce qui entraîne notamment des modifications dans les synthèses et un réagencement des cellules. L'identité du tissu obtenu dépend de sa détermination (Déterm. 1). La désagrégation stoppe réversiblement la progression autonome. Celle-ci apparaît donc comme la manifestation du comportement social élémentaire des cellules. B. L'induction. La progression autonome qui aboutit à la formation d'un tissu fait apparaître une période de compétence pendant laquelle la population est capable de s'engager dans une autre progression autonome et d'engendrer un autre tissu. La détermination ne se fait que si un contact s'établit avec une autre population de cellules qui joue le rôle d'inducteur, mais dont la présence n'est pas nécessaire au déroulement de la progression autonome qu'il fait démarrer. C. Le réajustement d'ensemble. Bien qu'engagées dans une même progression autonome, les cellules peuvent acquérir des «personnalités » très variées. Elles ne restent identiques que si elles enregistrent en même temps les mêmes informations extra-cellulaires. Ici, la population poursuit sa progression autonome dans un environnement hétérogène composé de deux tissus différents. Comme chaque cellule doit constamment réajuster ses activités à celles de ses voisines, la population, dans son ensemble, effectue un réajustement qui provoque la diversification des types cellulaires. Les nouveaux traits d'organisation qui apparaissent sont déterminés à la fois par le comportement social élémentaire particulier des cellules et par la composition de l'environnement de la population. 


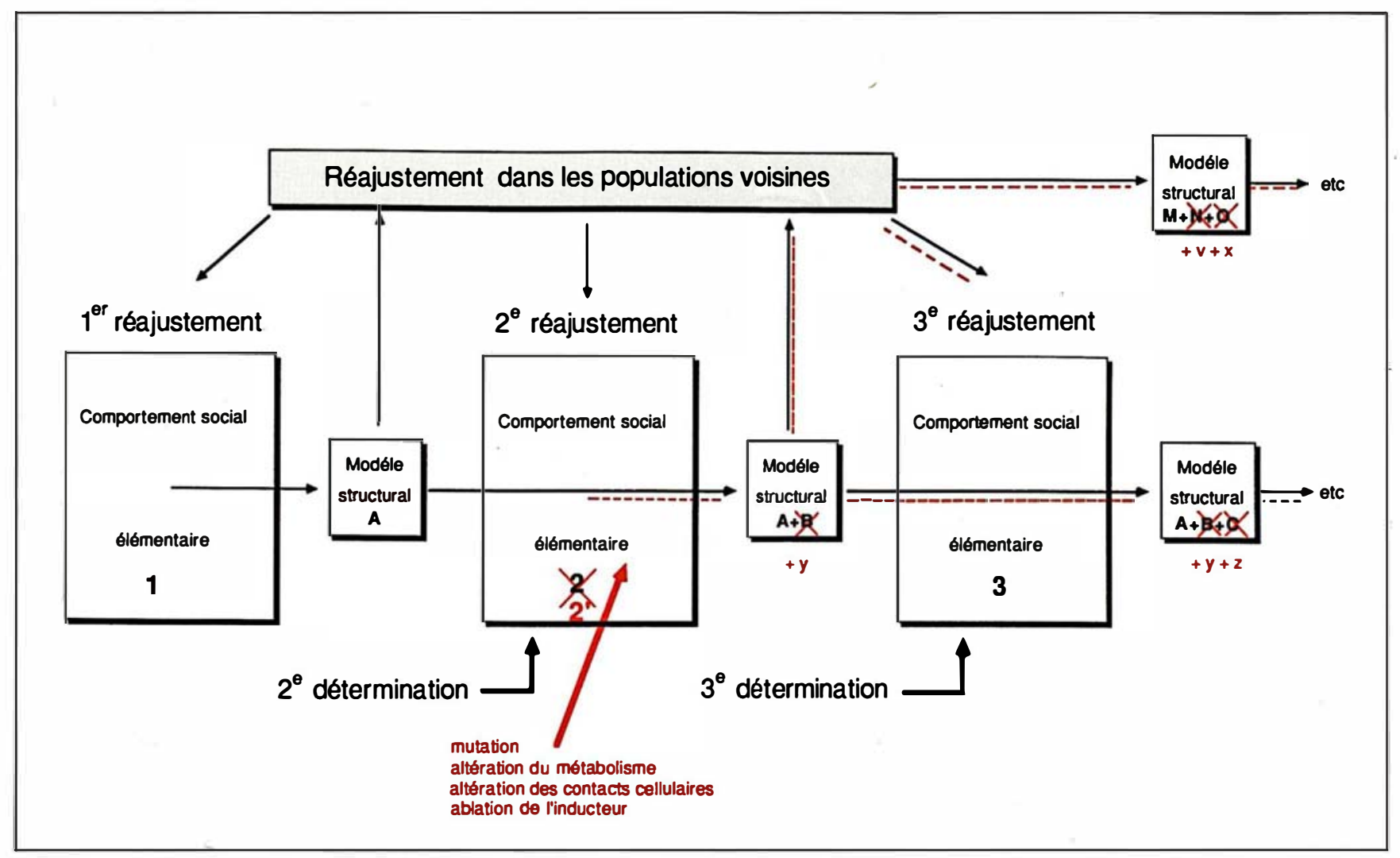

Figure 3. Schéma expliquant le mode d'action des facteurs tératogènes. En noir : les effets de sommation dans la reproduction des modèles structuraux au cours du développement normal. Dans chaque région de l'embryon, plusieurs déterminations sont nécessaires pour que les cellules parviennent à leur complète différenciation. Chaque détermination confère un comportement social élémentaire particulier aux cellules. Le réajustement particulier qui s'ensuit fait apparaître de nouveaux traits d'organisation et complique ainsi l'organisation acquise au cours du réajustement précédent. Cette complication se répercute sur les réajustements des populations voisines. En rouge: les effets des facteurs tératogènes. Par suite d'une mutation (génique ou chromosomique) ou de l'application d'un traitement tératogène, ou encore de la suppression d'un inducteur, les cellules qui s'engagent dans un nouveau réajustement prennent un comportement social élémentaire différent de celui qu'elles ont normalement à ce stade. Des traits anormaux s'introduisent. IIs falsifient les réajustements ultérieurs dans la descendance de cette population comme dans celle des populations voisines.

temps, puis se stabilise. Elle représente alors un tissu qui, chez l'embryon normal, apparaît à un stade ultérieur à celui du prélèvement (figure $2 A$ ). La désa régation peut entraîner un simple arrêt de la PA, jusqu'à ce que la réagrégation se produise, ou encore un retour en arrière, c'està-dire une dé-différenciation. La PA est l'œuvre de ce qu'on a appelé le «comportement social élémentaire» (CSE) des cellules [11]. La transformation des activités spécifiques entraîne celle de l'adhesivité différentielle, et par conséquent le réagencement $m / s n^{\circ} 3$ vol. 4, mars 88 des cellules. C'est pourquoi, pendant sa PA, une population change d'aspect histologique et, éventuellement, se déplace par rapport aux autres. Ainsi se mettent en place les traits les plus marquants dans l'organisation de l'individu, comme la segmentation du mésoderme, la disposition relative des feuillets embryonnaires, etc.

Au cours d'une PA, les cellules passent habituellement par une période de «compétence» pendant laquelle il leur est possible de s'engager dans une autre PA. La détermination s'effectue sous l'influence d'un tissu voisin qui joue le rôle d'inducteur (figure $2 B$ ). Ainsi se diversifient les activités tissulaires chez l'embryon. Comme elle impose seulement un choix entre deux voies possibles de différenciation, l'action de l'inducteur n'est pas spécifique. Elle est généralement reproductible par d'autres tissus (xéno-inducteurs) ou par de simples actions mécaniques qui modifient les contacts cellulaires. Dans un même tissu, les personnalités cellulaires sont diversifiables à l'infini. Au cours d'une PA, deux cellules ne peuvent rester 
identiques si elles ne recoivent pas en même temps des informations identiques de la part des cellules qui les entourent. Or, chez l'embryon, toute population évolue dans un environnement composite qui lui-même se transforme. Chacune des cellules doit constamment réajuster sa personnalité à celle des cellules de son entourage. Toute PA s'accompagne ainsi d'un « réajustement d'ensemble » (figure 2C) au cours duquel les types cellulaires se diversifient rapidement. La configuration des structures qui émergent (et qui sont maintenues grâce à la mémoire collective) est déterminée par le CSE particulier des cellules, d'une part, par les propriétés et les positions relatives des ébauches environnantes, d'autre part.

Le développement de n'importe quel organe implique plusieurs PA successives. A chaque détermination, les cellules changent de CSE et doivent effectuer un nouveau réajustement. Les nouveaux traits d'organisation qui apparaissent compliquent ceux que les réajustements précédents ont fait émerger (figure 3, page 171).

\section{Application à la tératogenèse}

Supposons qu'au moment où il met en place un certain trait d'organisation le CSE de certaines cellules soit altéré temporairement. Une anomalie structurale apparaît, d'abord anodine et très localisée. Mais elle falsifiera tous les traits d'organisation créés par les réajustements ultérieurs dans la descendance de ces cellules redevenues normales. Elle se répercutera aussi sur les tissus avoisinants (figure 3). La malformation ira en s'aggravant et sera d'autant plus sévère que l'accident s'est produit à un stade plus précoce. C'est pourquoi des mutations ou des traitements non mutagènes (produits chimiques, irradiations, chocs thermiques) peuvent avoir des effets tératogènes particuliers s'ils altèrent le CSE de certaines cellules à un
Un aspect du CSE qui retiendra plus particulièrement notre attention est la détermination de la vitesse à laquelle se déroule la PA. Son ralentissement ou son accélération, même momentanés, dans un tissu embryonnaire peuvent avoir des répercussions extrêmement graves sur le développement. En effet, dans ces cas-là, les cellules enregistrent les informations communiquées par leurs voisines trop tôt ou trop tard pour qu'elles soient correctement interprétées. Le déroulement du réajustement pourra s'en trouver faussé et ainsi une malformation apparaîtra, et ira en s'aggravant. Les répercussions peuvent être plus sévères encore si une compétence se manifeste avant ou après que l'inducteur soit prêt à assumer son rôle. L'absence d'organe entraîne des déficiences et des malformations dans les parties voisines, telles qu'on les observe après l'ablation chirurgicale de l'inducteur.

Or il est possible d'accélérer la PA dans un tissu embryonnaire par simple modification de la cohésion des cellules. Par exemple, le mésoderme somitique insegmenté, cultivé sous un film d'huile minérale, devient prématurément compétent pour donner du cartilage en présence de chorde [12]. Par ailleurs, les effets tératogènes de certaines mutations géniques ont pour origine l'accélération de la PA. Par exemple, chez les mutants Brachypod ${ }^{\mathrm{H}}$ de la souris, la différenciation progresse trop rapidement dans le mésenchyme du bourgeon de membre qui produit toutefois des îlots de précartilage normaux. Ce simple dérèglement suffit pour fausser l'organisation proximodistale de la patte[13]. Les effets tératogènes des anomalies chromosomiques - qui s'expliquent encore mal puisque les propriétés des gènes ne sont pas altérées pourraient également avoir pour origine l'accélération de la PA dans une ébauche [10]. En effet, d'après des données nouvelles, il semblerait que les modifications des propriétés cellulaires qui jalonnent la différenciation tissu- laire ne sont pas liées à l'activation de gènes jusque-là réprimés [14]. Chacune se produirait lorsque la concentration de certaines substances atteint un seuil critique. Il est ainsi possible que la PA soit accélérée dans un tissu embryonnaire si certains gènes sont représentés en trois exemplaires au lieu de deux.

\section{Un seul défaut dans l'ébauche de la chorde}

Chez les vertébrés, l'organogenèse débute au stade gastrula avec les trois « inductions primaires » $[15$, 16]. Au voisinage de l'ébauche de la chorde, la plaque neurale s'individualise dans l'ectoderme; le mésoderme différencie les somites (à l'origine des vertèbres) et les pièces intermédiaires (qui produisent le mésenchyme néphrogène); le tube digestif se met en place à partir de l'endoderme. L'émergence de ces nouvelles formations rend possibles toutes les autres inductions et réajustements ultérieurs, ce qui a valu à l'ébauche de la chorde le nom de « centre organisateur». Plus tard, celle-ci détermine encore l'émergence du bourgeon caudal, dont les somites engendrent le coccyx chez l'homme. Chez les vertébrés supérieurs, pendant la gastrulation, l'ébauche de la chorde, formée dans la partie antérieure de l'embryon, s'étire en direction postérieure, en clivant le mésoderme. Elle provoque sur son passage les trois inductions primaires.

Chez la sirène, l'enchaînement des événements ayant conduit aux malformations observées pourrait être le suivant: la gastrulation a commencé normalement et, de ce fait, aucun organe ne fait défaut dans les régions céphalique et thoracique. Mais l'extension de l'ébauche de la chorde a cessé prématurément, privant la région pelvienne des inductions primaires qui devaient s'y dérouler (figure 4). Les derniers somites du tronc ne se sont pas constitués, mais le bourgeon caudal a émergé normalement. La régulation étant ici impossible, l'anomalie a 


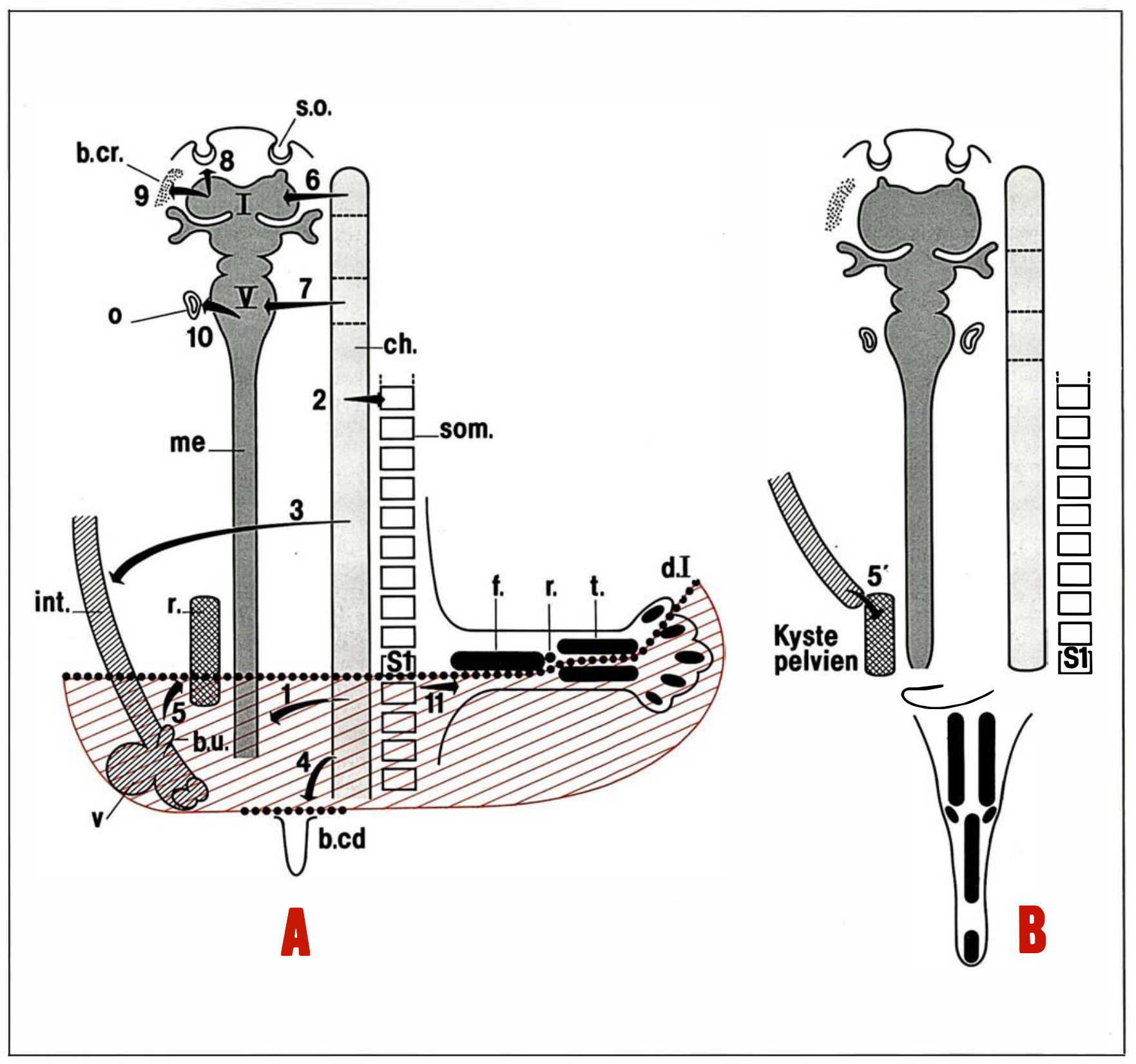

Figure 4. Comparaison de l'organisation de la sirène (B) avec celle du foetus normal (A). La partie recouverte de hachures rouges n'est pas développée chez la sirène. Les flèches noires montrent les principales inductions et interactions tissulaires du développement normal qui, chez la sirène, ont fait défaut ou se sont déroulées de manière anormale. 1. Induction de la moelle épinière (me) par la chorde (ch.) dont la région pelvienne a été privée. 2. Induction des somites (som.) par la chorde, également inachevée dans la région pelvienne. (S1: somite placé au niveau de la première vertèbre sacrée). 3. Induction de l'intestin (int.) et de ses dérivés: vessie (v) et bourgeons des uretères (b.u.). 4. Action de la chorde dans l'émergence du bourgeon caudal (b.cd). 5. Induction des reins (r) par les bourgeons des uretères. 5. Induction des kystes pelviens par de l'endoderme en situation ectopique. 6. Induction du télencéphale (I) par la partie antérieure de l'organisateur de l'encéphale. 7. Induction du rhombencéphale N) par la partie postérieure de l'organisateur de l'encéphale. 8. Induction des sacs olfactifs (s.o.) par le télencéphale. 9. Induction de la boîte crânienne (b.cr.) par le télencéphale. 10. Induction de l'otocyste (o) par le rhombencéphale. 11. Action des somites sur la différenciation du mésenchyme du bourgeon des membres (f. : fémur ; r. : rotule ; $t$. : tibia ; $d$. I: hallux). (Explications dans le texte.) 


\section{RÉFÉRENCES}

7. Wolpert L. Positional information and the spatial pattern of cellular differentiation. J Theor Biol 1969 ; 25 : 1-47.

8. Chandebois $\mathbf{R}$. Cell sociology and the problem of position effect, origin and role of gradients. Acta Biotheor 1977; 26 : 203-38.

9. Chandebois R, Faber J. Automation in animal development. A new theory derived from the concept of cell sociology. Monogr Dev Biol vol. 16. Bâle: Karger, 1983.

10. Chandebois R, Faber J. From DNA transcription to visible structure: what the development of multicellular animals teaches us. Acta Biotheor 1987 ; 36 : 61-119.

11. Ede DA. Cell behaviour and embryonic development. Int J Neurosci 1972 ; 3 : 165-74.

12. Ellison ML, Ambrose EJ, Easty GC. Chondrogenesis in chick embryo somites in vitro. J Embryol Exp Morphol 1969 ; 21 : 331 40.

13. Owens EM, Sorlush M. Accelerated maturation of limb bud mesenchyme by the Brachypod ${ }^{\mathrm{H}}$ mouse mutation. Differentiation 1983 ; 24 : 145-8.

14. Clayton RM. The molecular basis for competence, determination and transdifferentiation : a hypothesis. In : Clayton RM, ed. Stability and Switching in Cellular Differentiation. New York: Plenum, 1982 : 23-38.

15. Chandebois $\mathbf{R}$. Morphogénétique des animaux pluricellulaires. Paris: Maloine, 1976.

16. Nieuwkoop PD, Johnen AG, Albers B. The epigenetic nature of early chordate development. Inductive interaction and competence, vol. 16. Developmental and Cell Biology. New York: Cambridge University Press, 1985.

17. Ede DA, Agerbak GS. Cell adhesion and movement in relation to the developing limb pattern in normal and talpid ${ }^{3}$ mutant chick limb mesenchyme cells. J Embryol Exp Morphol $1968 ; 20: 81-100$.

18. Fujimoto $H$, Yanagisawa KO. Defects in the archenteron of mouse embryos homozygous for the $T$-mutation. Differentiation 1983 ; 25 : 44-7.

19. Cuny R, Malacinski GM. Axolotl retina and lens development: mutual tissue stimulation and autonomous failure in the eyeless mutant retina. J Embryol Exp Morphol 1986 ; $96: 151-70$.

20. Chandebois $\mathbf{R}$. The importance of embryology in the discussion of the problems of evolution. In : Mlikovský J, Novák VJA, eds. Evolution and Morphogenesis. Prague: Czeckoslovak Academy of Sciences, 1985: 4363.

21. Chandebois $\mathbf{R}$, Condamine $\mathbf{H}$. Débat sur la notion de programme génétique de développement. médecine/sciences 1986 ; 2 : 514 - entraîné une solution de continuité dans le rachis au niveau de la région sacrée. Rapprochées par suite de cette déficience, les ébauches des membres pelviens ont fusionné, ce qui a entravé les flexions et les torsions dont elles sont ultérieurement le siège. Ainsi ont été faussées la position des rotules, l'orientation du pied et celle des muscles abdominaux. Faute de somites au niveau de la moitié postérieure des bourgeons des membres pelviens, les pièces postérieures du squelette ne s'y sont pas différenciées. La moelle épiniere a récupéré une longueur normale, probablement grấce à une régulation secondaire. Son extrémité est restée effilée parce que les somites sont indispensables à la différenciation des pyramides. La présence des somites étant également requise pour le développement des nerfs rachidiens, leur nombre est déficitaire dans la queue de cheval. Le segment terminal de l'intestin, lui non plus, n'a pas été induit, ce qui explique l'absence de rectum, de vessie et d'uretères. A la place de ces derniers, l'épithélium intestinal aura probablement fait office de «xéno-inducteur». Il aura évoqué la formation de tubules uninifères observés dans les kystes pelviens. Ceux-ci sont restés à l'état embryonnaire parce que leur différenciation ne pouvait pas se poursuivre en l'absence d'uretères. L'extension incomplète de l'ébauche de la chorde s'explique par un arrêt prématuré de la migration de ses cellules, conséquence d'une PA trop rapide. Ce défaut n'a pas altéré leurs autres propriétés et notamment leur pouvoir inducteur.

La partie antérieure de la chorde joue le rôle d'organisateur céphalique $[15,16]$. Après avoir induit la plaque neurale, elle y imprime sa propre organisation, fixant ainsi les limites des territoires présomptifs de la moelle épinière et des vésicules de l'encéphale. Le télencéphale induit ensuite les sacs olfactifs et participe plus tard à la détermination de la boîte crânienne. Le rhombencéphale induit l'otocyste (figure 4). Chez la sirène, toutes les malformations observées dans la tête pourraient être attribuées à l'organisation défectueuse de la future chorde en direction rostro-caudale [3]. La partie qui induit le télencéphale a pris une extension trop importante (d'où l'hyperdéveloppement de la boîte crânienne et des fosses nasales), décalant vers l'arrière celle qui induit le rhombencéphale, d'où l'implantation basse de l'oreille. Les malformations céphaliques semblent avoir la même cause première que les malformations pelviennes, car nous les avons également observées chez les deux autres sirènes que nous avons eu l'occasion d'étudier. Effectivement, nos connaissances actuelles des interactions cellulaires impliquées dans l'édification du centre organisateur autorisent à penser qu'une telle dystrophie résulterait elle aussi de l'accélération de la PA chez les cellules déterminées en chorde (voir modèle théorique, figure 5).

En définitive, la cause première de la sirénomélie chez ce fotus au caryotype normal a pu être l'action de l'un des trois facteurs capables de provoquer l'accélération d'une PA, c'est-à-dire soit une mutation génique, soit une anomalie chromosomique inframicroscopique, soit un simple incident mécanique. Cette dernière possibilité retient plus particulièrement l'attention. En effet, au stade où elle commence à jouer son rôle de centre organisateur, l'ébauche de la chorde s'encastre dans l'endoderme (figure 6). Une éventuelle surpression dans le liquide du lécithocœle peut contraindre les cellules à s'étirer, d'autant plus aisément qu'elles sont en période de migration active.

\section{Intérêt théorique de la monstruosité}

Pour un certain nombre de mutations géniques, on connaît le dysfonctionnement cellulaire (locomotion lente [17], propriétés anormales du matériel intercellulaire [18]) qui introduit la malfor- 

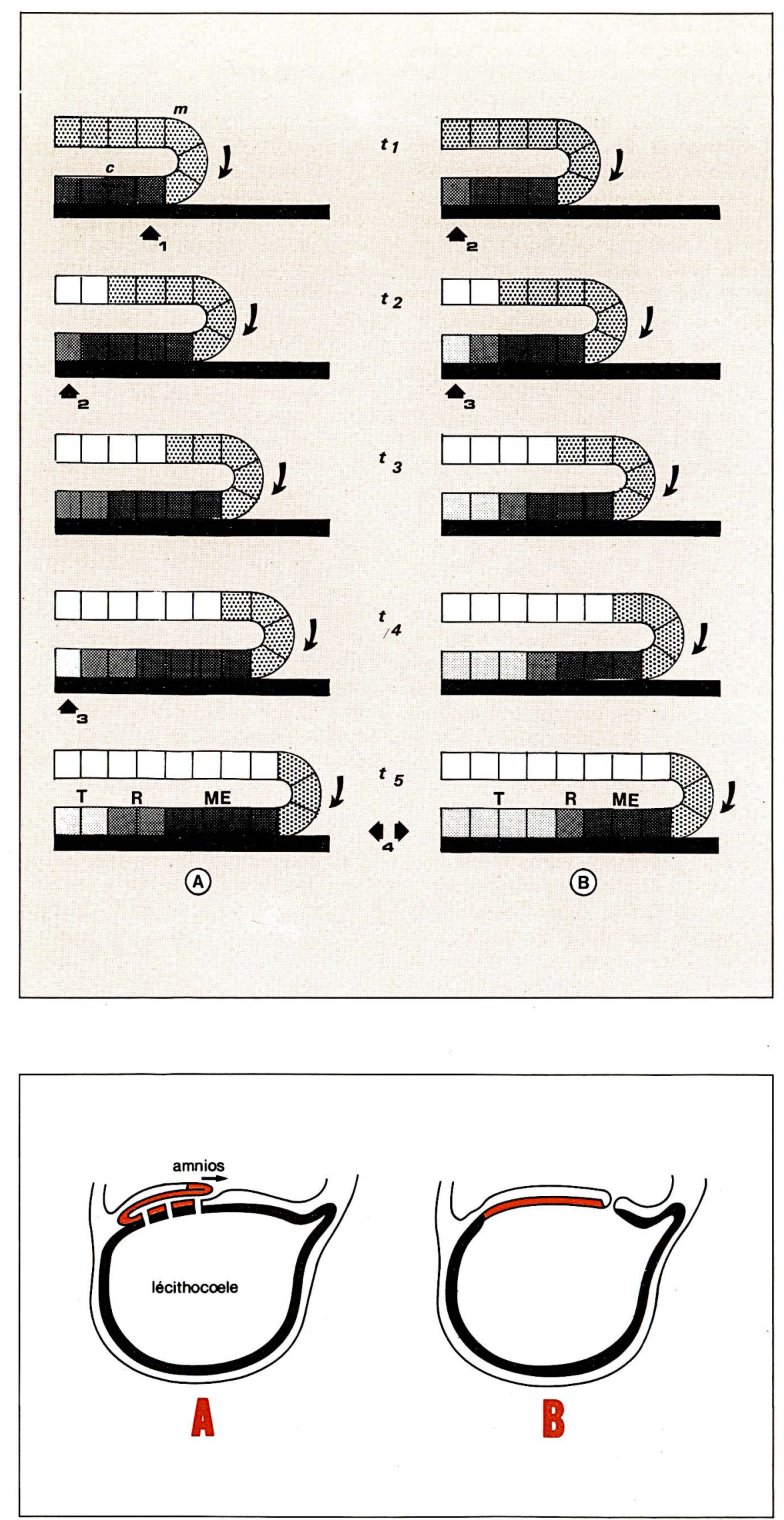

$m / s n^{\circ} 3$ vol. 4, mars 88
Figure 5. Représentation schématique de l'installation de l'organisation antéro-postérieure dans I'éb a u che de la chorde. A. Développement normal. Des cellules du feuillet superficiel ont été orientées vers la différenciation mésodermique $(m)$. Elles migrent en profondeur. Chaque fois que l'une d'entre elles entre en contact avec l'endoderme (en noir) elle est déterminée (flèche 1) pour donner de la chorde (c). A ce stade, les cellules sont capables d'induire la moelle épinière. Les cellules restent réceptives à l'action de l'endoderme jusqu'à l'extinction de leur compétence (flèche 4). Entre temps, elles acquièrent la propriété d'induire le rhombencéphale (flèche 2) puis, au bout d'un certain temps, celle d'induire le télencéphale (flèche 3). Les cellules les plus antérieures, qui sont les premières à avoir immigré, sont les seules qui sont restées assez longtemps sous l'influence de l'endoderme pour pouvoir induire le télencéphale (T). En arrière, les cellules plus récemment déterminées en chorde induisent la moelle épinière (ME). Entre les deux, les cellules qui induisent le rhombencéphale (R). B. Développement de la sirène. Après leur détermination, les cellules appelées à former le mésoderme axial accélèrent leur progression autonome. Leurs transformations ultérieures s'effectuent plus tôt laux stades 1 et 2 au lieu de 2 et 4). En conséquence, I'organisateur du cerveau est plus étendu que la normale et l'inducteur du télencéphale y tient une place relativement trop importante.

Figure 6. Coupes sagittales dans lembryon humain à deux stades de la gastrulation. A. Rupture du canal chordal (en rouge) pendant son extension en direction caudale. $B$. Ébauche de la chorde (en rouge) encastrée dans l'endoderme (en noir). 
mation, ainsi que les interactions cellulaires qui l'aggravent par la suite [19]. Notre sirene est un cas bien plus instructif. Elle devrait devenir un excellent modèle pour l'enseignement de la tératogenèse puisque les nombreuses inductions supprimées ou faussées comptent parmi les sujets les plus classiques de l'embryologie expérimentale.

Selon les vues courantes, la gravité des malformations chez la sirène évoque l'altération d'une grande partie du génome. En effet, on admet habituellement que le maintien d'un trait d'organisation exige l'entretien de la transcription sur un ou plusieurs gènes particuliers, réprimés ailleurs - exactement comme l'entretien de la fonction spécifique d'un tissu exige l'activité de séquences uniques de l'ADN, réprimées dans les autres tissus. Or on sait que, pour corriger les effets d'une mutation à l'aide d'un traitement non mutagène, il faut des stratégies différentes selon que l'on a affaire à un trouble physiologique ou à des malformations. Dans le premier cas, le traitement peut être administré avec succès à n'importe quel moment, mais il doit être maintenu pour obtenir des effets durables. Dans le cas des malformations, il doit être appliqué au moment même où l'anomalie s'introduit dans le développement, et ses effets sont définitifs. La raison de cette différence, c'est qu'une mutation aux effets tératogènes touche des propriétés cellulaires généralement banales (altérables par les agents tératogènes les plus divers) au moment où celles-ci sont impliquées dans la genèse d'un nouveau trait d'organisation. La spécificité de la malformation dépend des propriétés uniques des cellules déréglées par la présence d'une protéine atypique (ou par le facteur tératogène) et plus encore du caractère unique, dans l'histoire du développement, de l'enchaînement des interactions cellulaires touchées. Ainsi, avec un même accident dans la PA, la partie anormalement (ce qui fausse les réajustements dans la tête), tandis que la partie postérieure cesse son extension (d'où les déficiences dans la région pelvienne).

La plupart des traits faussés par l'activité d'un gène muté sont de nature «épigénétique ». Il faut entendre par là que les cellules ont joué incorrectement leur rôle, non pour avoir elles-mêmes utilisé les produits de ce gène, mais parce que les informations dont elles ont disposé étaient incorrectes ou incomplètes. Si la sirène est un mutant, toutes les malformations relevées à l'autopsie sont de nature épigénétique, puisque la chorde a dégénéré avant la fin de l'organogenèse. En raison des interactions cellulaires, chez un mutant, l'ampleur de l'anomalie est sans rapport avec l'importance de l'altération du génome mais dépend du rôle que les cellules touchées jouent dans le développement. Si, avec le même type de dysfonctionnement, une sirène est plus gravement atteinte qu'une souris Brachypod $^{\mathrm{H}}$, c'est parce que la chorde est le centre organisateur de l'embryon. L'importance théorique de cette remarque dépasse le cadre de la tératogenese. Elle justifie la possibilité des «saltations », c'est-àdire l'apparition brusque d'espèces nouvelles dans l'evolution des êtres organisés, puisque, par suite d'une simple mutation ponctuelle, un individu peut présenter une morphologie profondément différente de celle de ses progéniteurs [20].

On voit, à travers l'exemple de cette sirène, l'importance que devrait revêtir la connaissance des propriétés cellulaires en jeu dans la construction de l'embryon. Si cette voie de recherche est délaissée au profit de l'analyse de l'ADN, on aboutit obligatoirement à attribuer au matériel génétique une complexité structurale supérieure à celle qu'il possède en réalité. L'idée que l'organisation de l'être pluricellulaire est codée dans la séquence des nucléotides de l'ADN suscite actuellement quelque scepticisme [21]. L'exemple de notre sirène ne peut que le renforcer

\section{Summary}

Malformations and deficiencies observed in a foetus with sirenomelia are similar to those produced in vertebrate embryos upon various surgical operations performed on purpose to unravel cell and tissue interactions during development. From this comparison we inferred that the stretching backwards of the notochordal anlage has been arrested before the proper time. Consequently the pelvic region was deprived of primary and secondary inductions required for its normal development. Moreover the anteroposterior organisation of the cranial part of the notochordal anlage was abnormally laid out. This resulted in the dystrophy of the head. Finally these defects may be attributed to a single cause: the speeding-up of the autonomous progression of differentiation in the chordomesoderm which has been brought about either by a point mutation (the caryotype is normal) or by an excessive pressure in the yolk sac. In this paper sirenomelia is set up as a model illustrating a new theory of teratogenesis.

\section{TIRÉS A PART}

R. Chandebois : laboratoire de morphogénétique animale, université de Provence, centre Saint-Charles, place Victor-Hugo, 13331 Marseille Cedex 3, France. 